\title{
Mechanical thrombectomy in a young stroke patient with Duchenne Muscular Dystrophy
}

Spicher $C^{1}$, Schneider $R^{1}$, Mönnings $P^{2}$, Schneider-Gold $C^{1}$,
Kallenberg $D^{3}$, Cevik $B^{3}$, Lukas $C^{2}$, Gold $R^{1}$, Krogias $C^{1}$
Dept. of Neurology ${ }^{1}$, Dept. of Radiology ${ }^{2}$, and Dept. of Anästhesiology ${ }^{3}$
St. Josef-Hospital, Ruhr University Bochum, Germany

\begin{tabular}{l} 
Background: \\
Duchenne muscular dystrophy \\
(DMD) is an X-linked recessive \\
skeletal muscle myopathy which is \\
caused by mutations in the \\
dystrophin gene. Lack of dystrophin \\
also results to cardiomyopathy, \\
which raises significantly the stroke \\
risk in DMD-patients. However, data \\
about therapeutic opportunities in \\
the acute setting are scarce in \\
literature. So far, only two cases \\
receiving IV thrombolysis are \\
described, one of them with fatal \\
outcome. \\
We report of a case of successful \\
mechanical thrombectomy (MTE) in \\
an acute ischemic stroke (AIS) \\
patient with DMD and associated \\
dilatative cardiomyopathy. \\
Literature: Bushby K, Finkel R, Birnkrant DJ et al.: \\
Diagnosis and management of Duchenne muscular \\
dystrophy. Lancet Neurol 2010; 9: $77-93$ \\
Endovascular reperfusion therapies for acute \\
ischemic stroke: dissecting the evidence. \\
Expert Rev Neurother 2016;16:527-534. \\
\hline
\end{tabular}
Case Report:
A 20-year old DMD-patient was transferred at 08:56 $\mathrm{h}$ to our department due to wake up stroke with severe right-sided hemiparesis and aphasia (NIHSS=20). Last-seen-normal was at 03:00 h. Cerebral CT-scan revealed only slight early ischemic changes (ASPECT-Score $=8$ ). CT-angiography detected occlusion of left middle cerebral artery (LMCA). MTE started rapidly at 9:23 $\mathrm{h}$ and using direct thrombus aspiration (Penumbra System $\AA$ ) complete recanalization was achieved 20 min later ( $\mathrm{TICl}$-grade 3). Considering the specific risks of general anesthesia in DMD, the procedure was performed with propofol, remifentanil and rocuronium. The patient recovered quickly from the acute symptoms, due to preexisting hypotonic tetraparesis his NIHSS-score at discharge was 12 points.

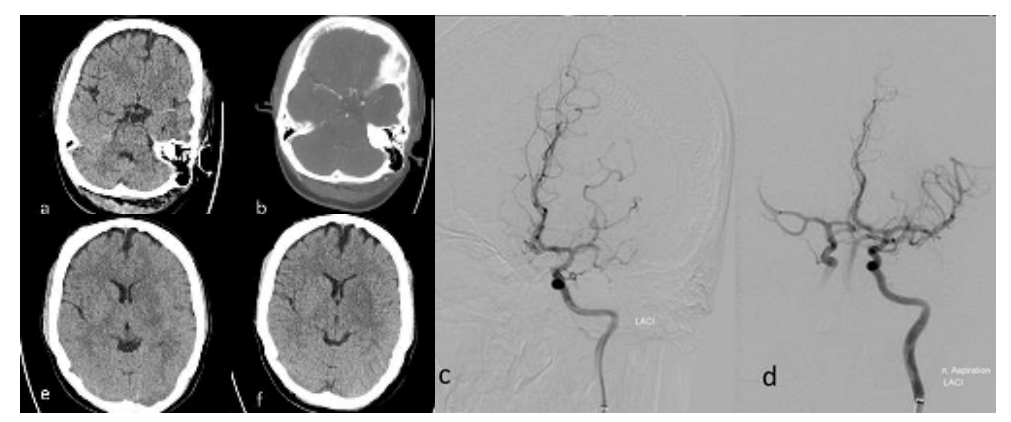

Initial CT-scan showing the hyperdense media-sign (1a), proximal occlusion of LMCA (1b), MT before aspiration of the thrombus (1c) and after aspiration (1d). Follow-up CT-scans showing a hypodensity in the left area of basal ganglia with a mild edema (1e and $1 \mathrm{f}$ ).

\section{Conclusion:}

To the best of our knowledge, this is the first report on MTE in a patient with DMD related cardioembolic stroke. In contrast to the few reports with IV thrombolysis, MTE seems to represent an optimal treatment option. Specific characteristics of DMDpatients like anesthetic regimen should be taken into account. 